\title{
Optical Clocks Join the Hunt for Dark Matter
}

\author{
Researchers use precise clocks and an extremely stable optical cavity to \\ find new constraints on how the dilaton, a hypothesized dark matter \\ particle, interacts with ordinary matter.
}

\section{By Sophia Chen}

( ptical clocks and optical cavities can today measure time and frequency to a precision of 1 part in $10^{18}$. Some researchers have begun to apply these precise measurement capabilities to look for physics beyond the standard model. Now, Colin Kennedy and colleagues at JILA in Colorado have used an optical clock, a microwave clock, and an exceptionally stable optical cavity to look for an extremely light dark matter particle called a dilaton [1]. This particle is predicted by some extensions of the standard model, including string theory. While the team does not confirm the dilaton's existence, their measurements expand constraints on its interaction with ordinary matter.

Interactions between a dilaton and regular matter are predicted to manifest as tiny fluctuations in the apparent value of the fine structure constant and in the mass of the electron. Kennedy and his colleagues looked for these fluctuations by comparing the resonant frequency of a silicon optical cavity to the ticks of

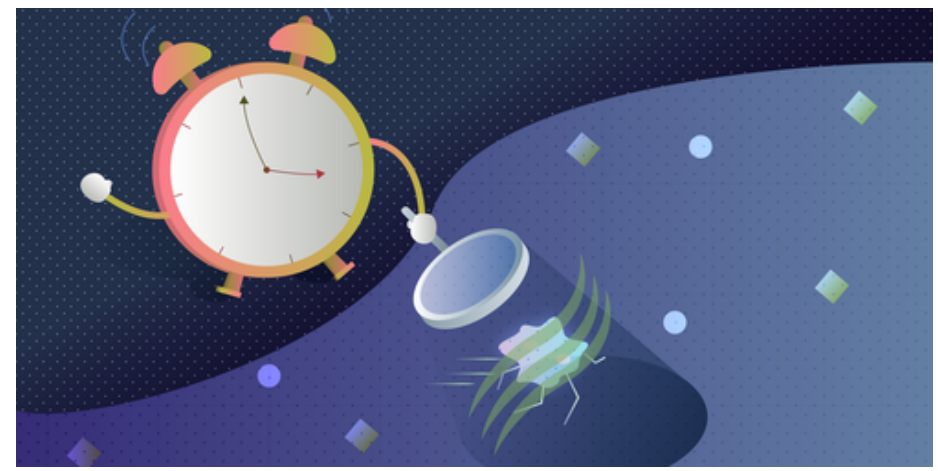

Credit: Hanacek/NIST atomic clocks.

Specifically, they compared the optical cavity's resonant frequency to the frequency of the atomic transition in a strontium optical clock and in a hydrogen maser clock. Each source produces a frequency of light that depends differently on the fine structure constant and on the electron mass, allowing the team to use the frequency comparison to study potential variations in the constants. They show that their measurements constrain the dilaton's interactions with ordinary matter by up to 2 orders of magnitude for certain dilaton-mass ranges.

The technique represents a new way to probe fundamental constants using optical frequency standards. Thus, the researchers say that their work motivates the building of a global optical-clock network for sensing physics beyond the standard model.

Sophia Chen is a freelance science writer based in Columbus, Ohio.

\section{REFERENCES}

1. C. Kennedy et al., "Precision metrology meets cosmology: Improved constraints on ultralight dark matter from atom-cavity frequency comparisons," Phys. Rev. Lett. 125, 201302 (2020). 